\title{
Laser-Machined Tantalum Collimator for Space Applications
}

\author{
M. Christophersen ${ }^{*}$, B. F. Phlips ${ }^{*}$, J. A. Christodoulides ${ }^{* *}$, R. S. Woolf ${ }^{* * *}$, and L. A. Jackson ${ }^{* * * *}$ \\ *U.S. Naval Research Laboratory, Code 7654, 4555 Overlook Ave. S.W. Washington, DC 20375, \\ USA \\ E-mail: marc.chrisophersen@nrl.navy.mil \\ ** U.S. Naval Research Laboratory, Code 6363, 4555 Overlook Ave. S.W. Washington, DC 20375, \\ USA \\ ${ }^{* * *}$ NRC Postdoctoral Fellow at the U.S. Naval Research Laboratory, Code 7654, 4555 Overlook Ave. \\ S.W. Washington, DC 20375, USA \\ ${ }^{* * * *}$ Praxis Inc., 5845 Richmond Highway, Suite 700, Alexandria, VA 22303, USA
}

\begin{abstract}
We are developing and prototyping tantalum X-ray collimators for LOFT (Large Observatory For X-ray Timing), a mission proposal selected by ESA as a candidate mission. LOFT is devoted to $\mathrm{X}$-ray timing and designed to investigate the space-time around collapsed objects. The collimator is micro-fabricated in a two-step process: (i) laser-machining of a hole array in Ta and (ii) chemical etching of the hole with the desired porosity (hole vs. bulk volume). The collimator design was studied by Monte Carlo simulation using SWORD (SoftWare for Optimization of Radiation Detectors). The anticipated collimator performance shows 2 orders of magnitude rejection over 2 degrees. The angular response of the collimator was measured with a commercial X-ray diffractor. The prototype collimates over $+/-5$ degrees.

DOI:10.2961/jlmn.2013.02.0012
\end{abstract}

Keywords: Laser-micromachining, X-ray collimator, LOFT mission, chemical etching of tantalum.

\section{Introduction}

LOFT, the Large Observatory For X-ray Timing, is a newly proposed space mission that will answer fundamental questions about the motion of matter orbiting close to the event horizon of a black hole, and the state of matter in neutron stars [1]. LOFT was recently selected by ESA as a mission candidate that will compete for a launch opportunity at the start of the 2020s. (AXTAR is another X-ray timing mission concept, currently under study in the U.S. [2]. The mission goals and requirements are, to a certain extend, similar to LOFT.)

LOFT is specifically designed to exploit the diagnostics of very rapid X-ray flux and spectral variability that directly probe the motion of matter down to distances very close to black holes and neutron stars. The LOFT Large Area Detector (LAD) has an effective area a factor of $~ 20$ larger than the largest area X-ray instrument ever flown (RXTE, Rossi X-ray Timing Explorer). LOFT/LAD's much improved energy resolution (better than $260 \mathrm{eV}$ ) compared to that of RXTE will also allow the simultaneous exploitation of spectral diagnostics, in particular the relativistically broadened 6-7 keV Fe-K lines. The timescales that LOFT will investigate range from sub-millisecond quasi-periodic oscillations (QPOs) to years long transient outbursts.

The LAD will be the main instrument for LOFT, see Fig (1). The overall LOFT instrument is mounted on 6 petals. The crucial characteristic of the LOFT Large Area Detector is a mass per unit surface in the range of $\sim 10$ $\mathrm{kg} / \mathrm{m}^{2}$ (for comparison, the largest predecessor, the RXTE instrument, has $>100 \mathrm{~kg} / \mathrm{m}^{2}$ ), enabling $\sim 20 \mathrm{~m}^{2}$ geometric area payload at reasonable weight. The ingredients for a sensitive but light experiment are the large-area silicon semiconductor X-ray detector, and a collimator. The current LAD design is based on lead-glass micro-capillary plates (the mechanical structure of the well-known microchannel plate). The target field-of-view (FOV) is 60 arcmin FWHM (Full Width Half Max) with an open fraction of $\sim 70 \%$ (hole vs. wall fraction). The FOV leads to a 60:1 aspect ratio for the holes of the collimator.

We are developing a new collimator technology, consisting of thin tantalum sheets with laser micro-machined holes that promises better X-ray attenuation when compared to conventional collimator technologies. The high $\mathrm{Z}$ (atomic number) of tantalum enables excellent performance at energies up to $80 \mathrm{keV}$, unlike alternatives that employ lead-glass micro-capillary plates. Although, the mass of the Ta is higher than lead glass.

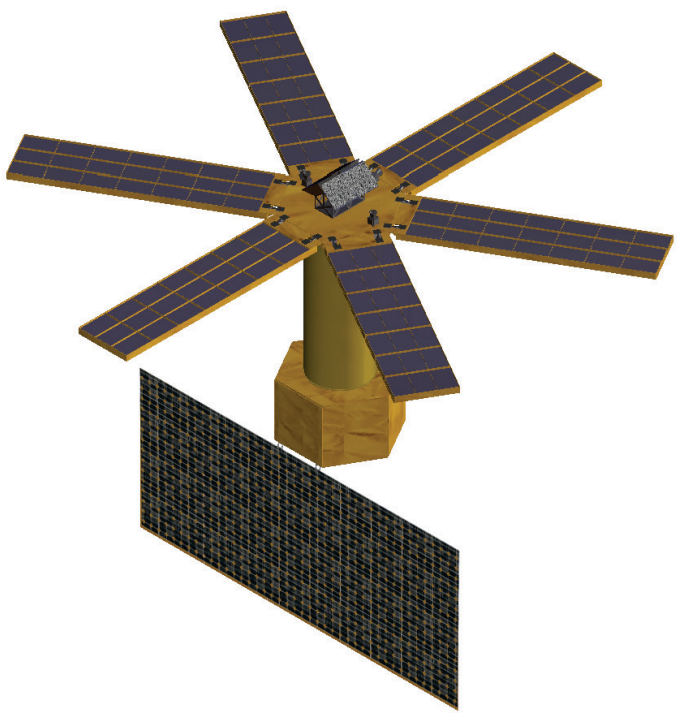

Fig. (1). Conceptual drawing of the LOFT mission. The detectors and collimators are on the six "petals". 


\section{Modeled Performance}

The expected performance of a tantalum collimator and lead glass collimator was modeled using Monte Carlo radiation transport programs. The SWORD software package [3] was used to model a micro-machined collimator in GEANT4 [4]. The same collimator geometry was modeled with two different materials, tantalum and lead glass $(40 \%$ lead by weight, or a density of $\left.5.9 \mathrm{~g} / \mathrm{cm}^{3}\right)$. The modeled geometry consisted of a $2 \mathrm{~mm}$ thick collimator with 53 micron holes on a 60 micron pitch hexagonally close packed. A total of $\sim 15,000$ holes were modeled to simulate $\mathrm{a} \sim 7 \mathrm{~mm} \times 8 \mathrm{~mm}$ collimator area.

\subsection{Lead-glass collimator}

The angular response was modeled in 0.1 degree steps for 10, 20, 40 and $80 \mathrm{keV}$ beams of photons. The results are shown in Fig. (2). The poor collimation of the lead glass at energies of $20 \mathrm{keV}$ (and higher) is very clearly seen.

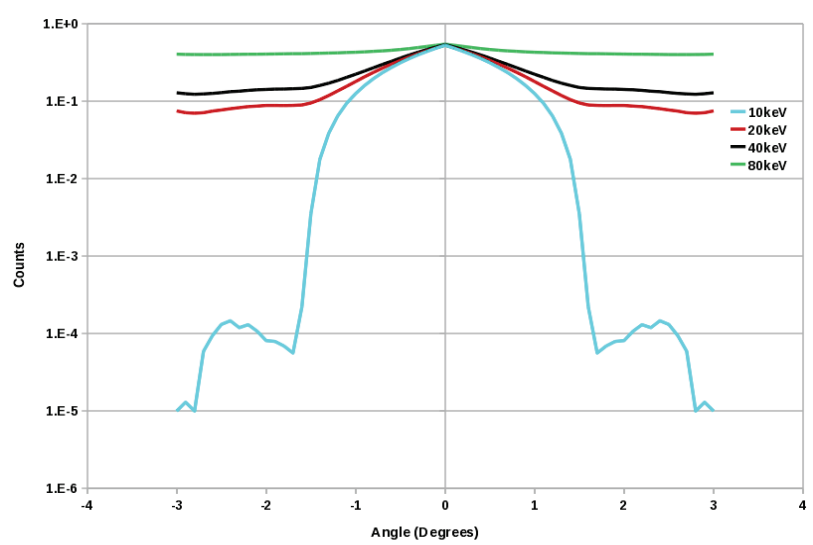

Fig. (2). Simulated angular response of lead glass collimator.

\subsection{Tantalum collimator}

The simulation of the tantalum collimator shows that even at $40 \mathrm{keV}$, the maximum first side lobe (at $\sim 2.5 \mathrm{de}-$ grees) is attenuated by more than two orders of magnitude, see Fig. (3). This is achieved with a $2 \mathrm{~mm}$ thick collimator.

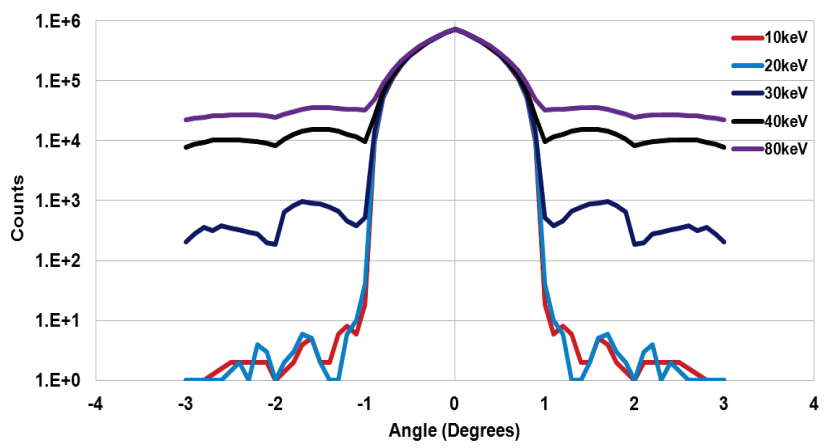

Fig. (3). Simulated angular response of lead glass collimator.

\section{Collimator micro-fabrication}

We used a turn-key laser micro-machining system from Oxford Laser, E-Series, for the micro-fabrication of the collimator prototypes. The E-Series has a diode-pumped solid state, frequency-tripled Nd:YAG laser (355 nm wave- length). The laser pulse was set at $10 \mathrm{~mJ}$ with a pulse duration of $50 \mathrm{~ns}$. The pulse repetition rate was $10 \mathrm{KHz}$. The drilling time per hole varied depending on the Ta sheet thickness, see section 3.1. The E-Series from Oxford Lasers has a moveable the stage. The movement speed, from hole to hole, was set at $1 \mathrm{~mm} / \mathrm{s}$.

A two step process micro-fabrication is utilized to produce the high aspect ratio hole arrays: (i) laser-machining and (ii) a chemical etch step. Both steps are described below.

\subsection{Laser-machining}

The aspect ratio needed for the LOFT collimator is 60:1 with a minimal tantalum thickness of $\geq 2 \mathrm{~mm}$. We started with $2 \mathrm{~mm}$ thick Ta, in order to fabricate the collimator from a single sheet.

Since the laser intensity distribution along the hole axis has an influence on the taper, we first optimized the laser focus for deep percussion drilling in Ta. We found that the hole taper can be reduced by moving the focus point. We used four different focal spots per hole. The drilling time was divided equally for each spot. The first spot was the sheet top surface. The next three was successively deeper into the bulk, Fig. (3) shows a schematic.

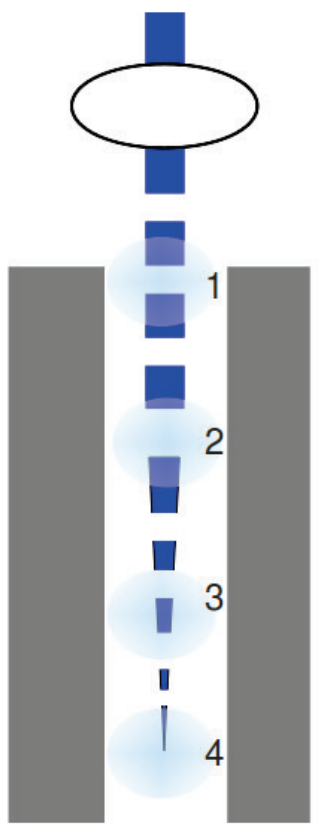

Fig. (4). Schematic of focus adjustment during percussion drilling, during drilling the focus point is moved from the sample surface (1) into the bulk material (2-3).

Our initial plan was to make the collimator from one sheet of Ta ( $2 \mathrm{~mm}$ thick, hole aspect ratio 60:1). Fig. (5) shows an optical micrograph from through hole in $2 \mathrm{~mm}$ thick Ta. The hole diameters for front and back side were $24.4 \mu \mathrm{m}$ and $17.45 \mu \mathrm{m}$ respectively. The corresponds to an aspect ratio of $\sim 85: 1$, what is sufficient for the collimator but the taper of $\sim 89.9$ degrees is not acceptable because it leads to $<70 \%$ open fraction after chemical etching. 


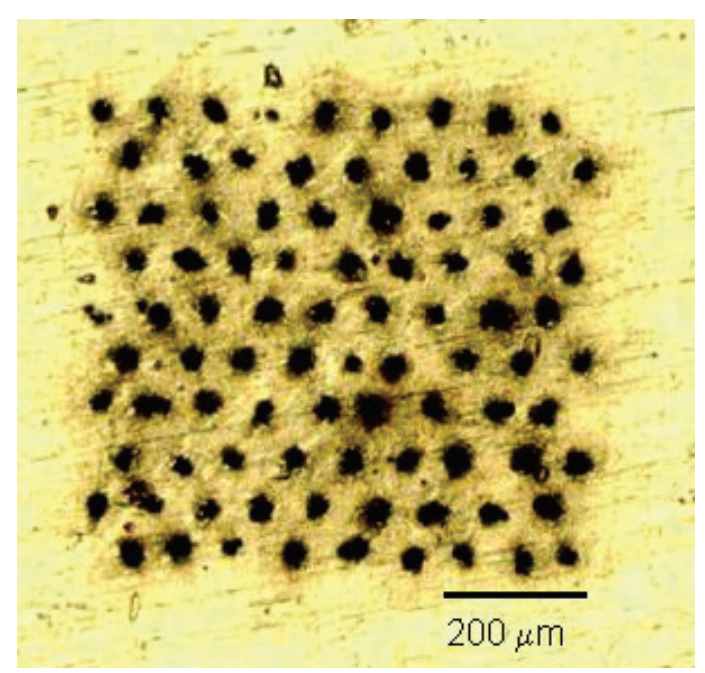

Fig. (5). Optical micrograph from through holes in $2 \mathrm{~mm}$ Ta.

Since we found a significant taper for $2 \mathrm{~mm}$ thick Ta sheets, we reduced the thickness of the sheets to $1.5 \mathrm{~mm}$. Fig. (6) shows optical micrographs from the front and back side from through holes in $1.5 \mathrm{~mm}$ thick Ta. The hole diameters are $17 \mu \mathrm{m}$ and $20 \mu \mathrm{m}$ for the back and front side. This corresponds to an aspect ratio of 80:1 and a taper of less than $<0.1$ degree.

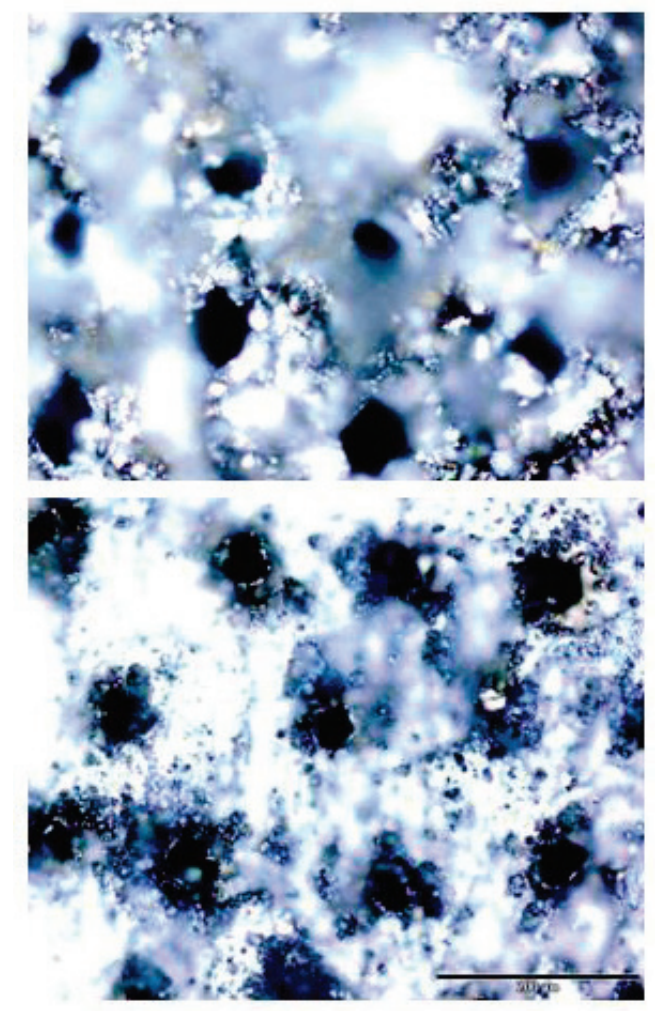

Fig. (6). Optical micrographs from through holes in 1.5 $\mathrm{mm}$ Ta, top front side and bottom back sidce (scale bar: 50 $\mu \mathrm{m})$.

The proposed collimator would be constructed out of 2 sheets of tantalum sandwiched together into a 2-3 mm thick collimator, see Fig. (7) for a schematic. Each sheet will have a hexagonal array of circular holes in it, which are micro-machined using a two step process of laser drilling and chemical etching. The resulting holes in each sheet have an aspect ratio of 30:1. The two plates have matching hole patterns and alignment pins that assure that the sets of holes are kept co-aligned. The full collimator thus has holes with a 60:1 aspect ratio, yielding a FWHM (Full Width Half Max) field of view of 1 degree.

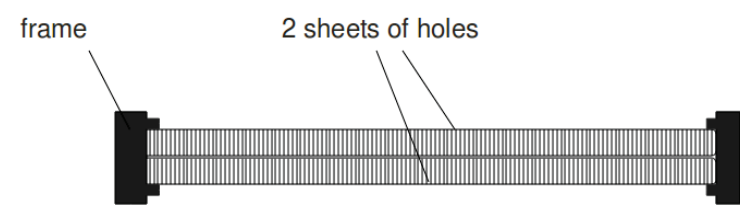

Fig. (7). Schematic of full collimator build from 2 sheets of Ta.

Although, a nitrogen gas flow and a vacuum suction for debris removal is part of the Oxford system, after lasermachining there is debris on the front side, see Fig. (8) for an optical micrograph. Fig. (8) was taken right after lasermachining - without any additional cleaning step. We found that careful scraping with a razor blade. The chemical etch step (next section) helps to clean up the front side by etching away debris particles.

We did not use any anti-spatter composite coating (ASCC). For future drilling tests we plan to use some type of ASCC for tantalum, in order to further reduce the surface debris.

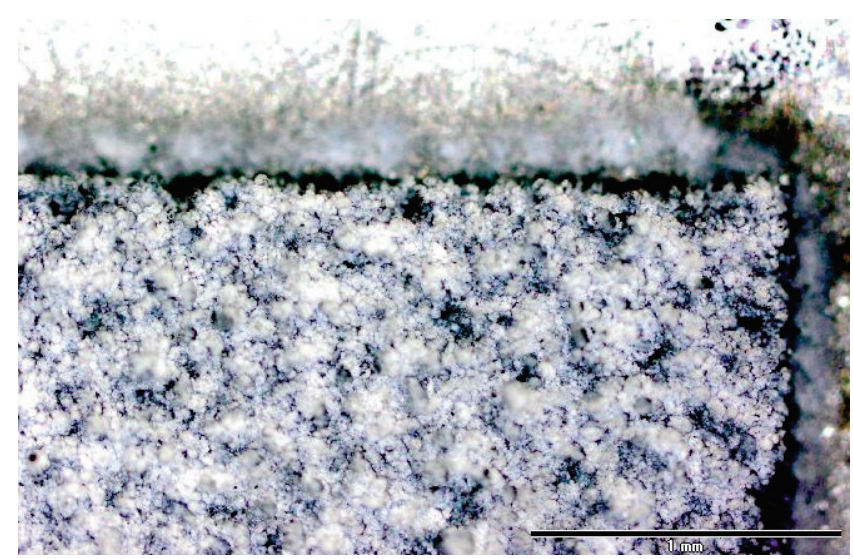

Fig. (8). Optical micrograph from surface debris (scale bar is $1 \mathrm{~mm})$.

\subsection{Chemical etching}

After laser-machining, the holes were reamed by a chemical etch step. The etch solution is a commercial product from Transene, Tantalum Etchants 111. Tantalum Etch 111 is a 1:1:1 mixture of HF (hydrofluoric acid), $\mathrm{HNO}_{3}$ (nitric acid) and water. The nitric acid oxidizes the tantalum and the hydrofluoric acid dissolves the generated oxide. The reaction is exothermic, generating heat. Large tantalum sheets need to be cooled during etching. Fig. (9) shows the etch rate of from Transene, Tantalum Etchants 111. 


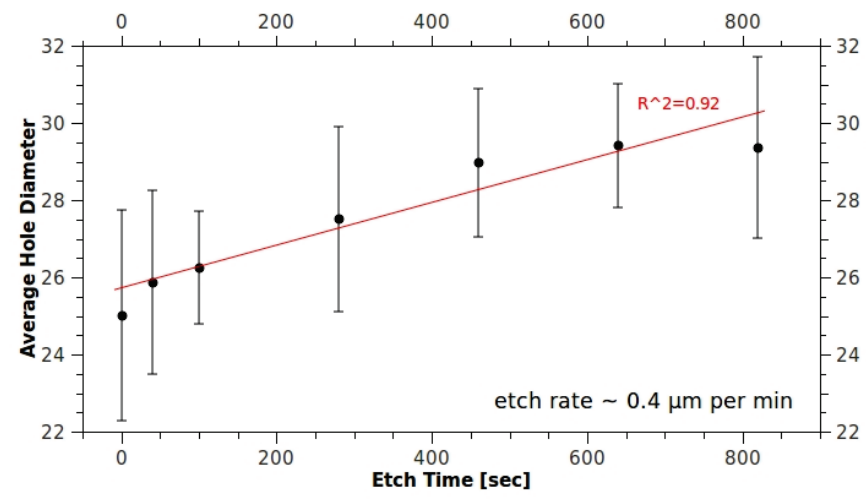

Fig. (9). Etch rate of Transene 111 at room temperature

The primary purpose of the etching step is to enlarge the holes to the desired diameter. Furthermore, the etching step allows for a fast laser-drilling because some of the material is removed during the chemical etch step. Fig. (10) shows an array before and after chemical etching.

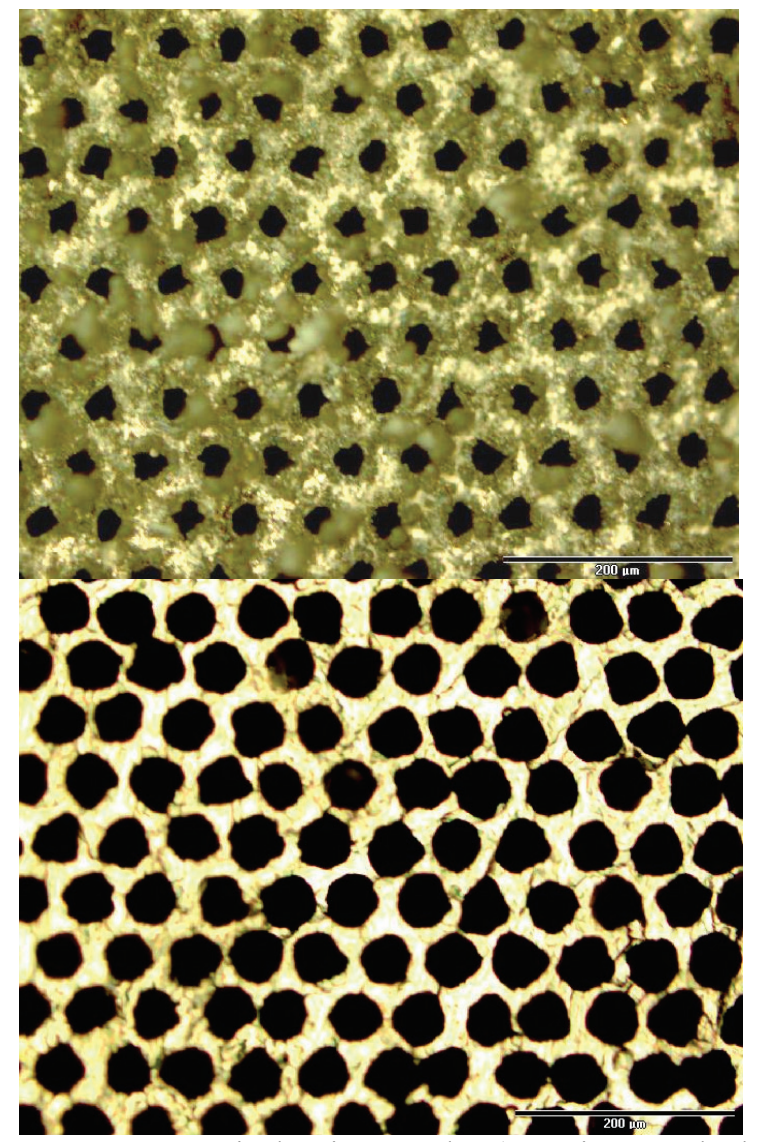

Fig. (10). Optical micrographs (top-view, scale bars $200 \mu \mathrm{m}$ ): before (top) and after (bottom) the chemical etch step.

There are several advantages for using a chemical etch step after laser-machining:

- The chemical etch removes the heat affected zone (HAZ).

- The chemical etch step enables the accurate adjustment of open fraction.
- The hole spacing not limited by heating of septum during laser-machining, the chemical etch step allows the fabrication of a thin septum.

- The chemical etch step removes some debris from the front side. The debris is tantalum based and can be etched. Transene 111 can etch Ta, TaN, and $\mathrm{Ta}_{2} \mathrm{O}_{5}$. Since the laser-machining is done in air. Tantalum can react with the air's oxygen and nitrogen.

\section{Large area machining}

One challenge is to maintain high quality holes (low taper, homogeneous diameter, etc.), while keeping the drilling speed high at economical costs for large areas. The target size of a collimator should match the unit size of the active area of each of the LOFT X-ray detector which is $110 \mathrm{~mm} \times 70 \mathrm{~mm}\left(7,100 \mathrm{~mm}^{2}\right)$.

Currently the largest prototype collimator has a size of $2,500 \mathrm{~mm}^{2}$, see Figs. (11-12). Since the laser stage is not $100 \%$ flat, during laser-machining the first focus point (sample surface) was adjusted to correct for the stage tilt.

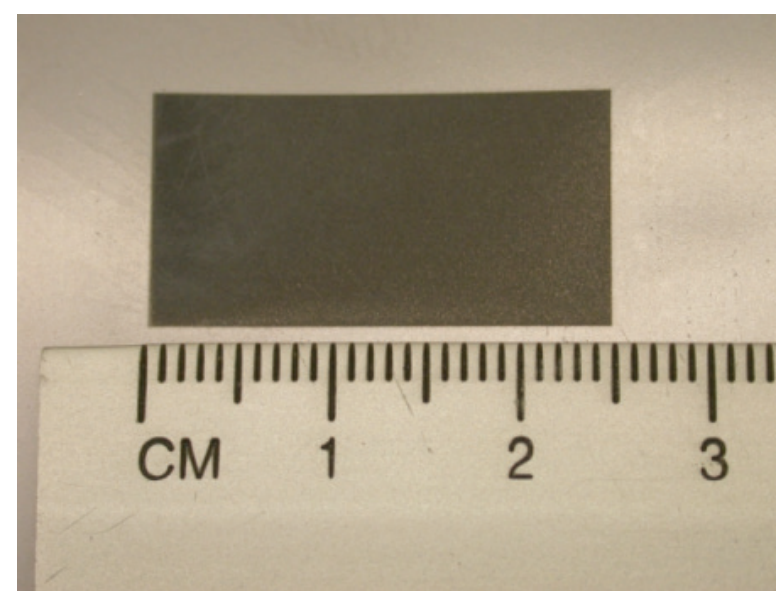

Fig. (11). Photo from large area prototype.

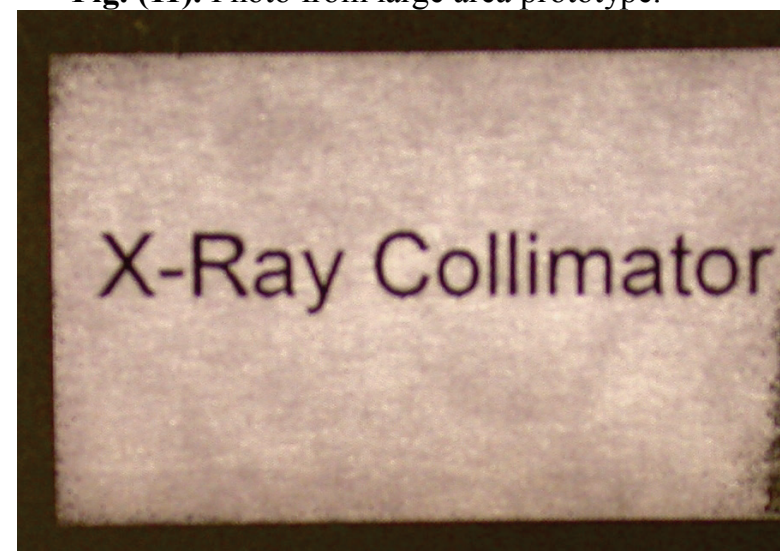

Fig. (12). Photo through collimator, back-lit paper, same prototype as seen in Fig. (11).

\section{Prototype testing}

The angular response of the collimator was measured with a commercial PANalytic Empyrean multi-purpose research diffractometer, see Fig. (13). Empyrean can perform preferred orientation analysis using the chi-tilt method (steps of $0.0001^{\circ}, 2 \theta$ linearity $\pm 0.01^{\circ}$ ). The X-ray energy was set at $20 \mathrm{keV}$. 


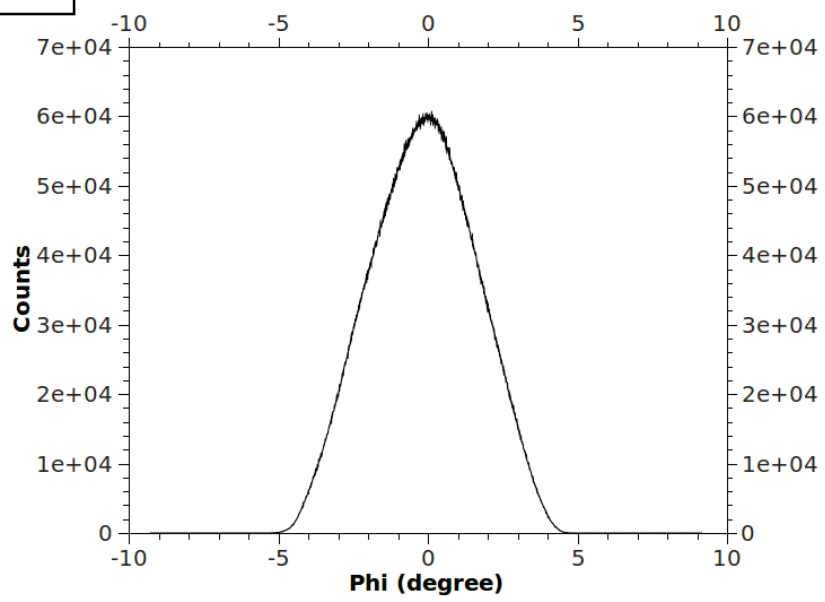

Fig. (13). Angular response of the prototype tantalum collimator measured at $20 \mathrm{keV}$, data in black, Gauss fit in red $(1 \mathrm{~mm}$ thick Ta sheet, $70 \mu \mathrm{m}$ hole diameter, same prototype as seen in Fig. (12)).

\section{Conclusions and outlook}

We present an X-ray collimator technology, consisting of tantalum sheets with laser-micromachined hole arrays. The high $\mathrm{Z}$ of tantalum enables excellent performance at energies up to $80 \mathrm{keV}$, unlike alternatives that employ leadglass micro-capillary plates.

In the future, we will investigate soldering or spotwelding of two tantalum sheets. Furthermore, we investigate the effect of an ASCC coating for the reduction of surface debris. We also plan to use Sequential Pulse Delivery Pattern Control (SPDPC). Hole taper control can be achieved by SPDPC using a linear increase in linearly increasing the laser pulse energy during laser percussion drilling [6].

\section{Acknowledgments}

We thank Dr. Paul S. Ray (U.S. Naval Research Laboratory) for helpful discussions and leading the U.S. LOFT group. Furthermore, we would like to thank the Institute for Nanoscience (NSI) at the Naval Research Laboratory (NRL) and the NSI staff members.

This work was funded in part by the Office of Navy Research (ONR).

\section{References}

[1] F. K. Lamb, "Scientific Challenges for a New X-ray Timing Mission," in X-ray Timing 2003: Rossi and Beyond, Kaaret, P., Lamb, F. K., and Swank, J. H., eds., AIP Conf. Ser. 714, (2004) 3.

[2] P. Kaaret, F. K. Lamb, and J. H. Swank, X-ray Timing 2003: Rossi and Beyond, AIP Conf. Ser. (2004) 714.

[3] D. Chakrabarty, P. S. Ray, and T. E. Strohmayer, "The Advanced X-ray Timing Array (AXTAR)", AIP Conference Series, 1068, (2008) 227.

[4] C. S. Gwon, C.S., et al. "Interacting with SWORD (SoftWare for the Optimization of Radiation Detectors)", IEEE NSS Conference Record (2007).

[5] Agostinelli, S., et al. "GEANT4--a simulation toolkit", Nucl. Instrum. Meth. A, 506 (3), pp 250-303, (2003).

[6] D. K. Y. Low, L. Li, P.J Byrd, "The influence of temporal pulse train modulation during laser percussion drilling”, Optics and Lasers in Engineering, 35 (2001) 149-164.

(Received: May 29, 2012, Accepted: March 14, 2013) 